\title{
On explicit relations between cyclotomic numbers
}

\author{
by
}

\author{
MARC CONRAD (Saarbrücken)
}

1. Introduction. For $n \in \mathbb{N}$ let $\varepsilon_{n}$ be a primitive $n$th root of unity and $D^{(n)}$ the multiplicative group generated by the elements $1-\varepsilon_{n}^{k}$ with $k \not \equiv 0 \bmod n \operatorname{modulo}$ roots of unity in order to avoid torsion. We call $D^{(n)}$ the group of cyclotomic numbers. This group and its subgroups, especially the group of cyclotomic units, have often been subject of investigation (see [1], [5]-[8], [11]). Here, we focus on the relations between the generators $1-\varepsilon_{n}^{k}$.

In $D^{(n)}$ there are two types of relations which we call the obvious relations.

- Relations arising by relative norms in cyclotomic fields. They can be deduced from the polynomial identity

$$
\prod_{\nu=0}^{p-1}\left(1-x \varepsilon_{p}^{\nu}\right)=1-x^{p}
$$

where $p$ is a prime, by inserting an appropriate root of unity for $x$.

- Relations arising by complex conjugation:

$$
1-\varepsilon_{n}=-\varepsilon_{n} \overline{\left(1-\varepsilon_{n}\right)}=-\varepsilon_{n}\left(1-\varepsilon_{n}^{-1}\right) .
$$

Surprisingly, Ennola [5] gave for $n=105$ a relation that is not generated by the obvious relations. In the following we will call such a relation an Ennola relation. Schmidt investigated in [8] the gap between the obvious relations and all relations. He connected this gap to cohomology groups which have been computed in [10].

Here, we will give explicit algorithms on how to construct all Ennola relations in $D^{(n)}$ for an arbitrary $n$. This will be done in a general context. We consider a free $\mathbb{Z}$-module $M$ with an involution $\sigma$ operating on it and introduce algorithms focusing on the construction of special bases of $M$, the so called $\sigma$-bases, that lead to generators of the torsion group of $M /(1-\sigma) M$

2000 Mathematics Subject Classification: 11R18, $20 \mathrm{~F} 05$. 
and $M /(1+\sigma) M$. Then we introduce a module $\mathcal{L}(n)$ for which we have by [2] an exact sequence

$$
0 \rightarrow T(n) \rightarrow \mathcal{L}(n) /(1-\sigma) \mathcal{L}(n) \rightarrow D^{(n)} \rightarrow 1
$$

where $T(n)$ is the torsion group of $\mathcal{L}(n) /(1-\sigma) \mathcal{L}(n)$. The relations in $(1)$ correspond to relations in $\mathcal{L}(n)$ and the relations in (2) correspond to the factorization by $(1-\sigma) \mathcal{L}(n)$. Thus, the Ennola relations are implicitly given in the torsion group $T(n)$, and the construction of a $\sigma$-basis of $\mathcal{L}(n)$ allows the construction of the elements of $T(n)$ explicitly.

In the last two sections we present similar results for the group of relative cyclotomic numbers and the Stickelberger ideal.

2. $\sigma$-bases. In the following all modules are finitely generated $\mathbb{Z}$-modules. When we speak of a basis we mean a $\mathbb{Z}$-basis. We assume further that an involution $\sigma$ operates on each module. The homomorphisms between two modules will always be compatible with the action of $\sigma$. So, we will have in fact $\mathbb{Z}[\sigma]$-modules and $\mathbb{Z}[\sigma]$-homomorphisms.

For a set $B$, a module $M$ and a mapping $\xi: B \rightarrow M$ we say that $B$ induces a basis of $M$ if the set $\{\xi(b): b \in B\}$ is a basis of $M$. Saying that some set is a basis of $M$ includes that $M$ is free.

A $\sigma$-basis of a module $M$ is defined as a triple $\left[E^{0}, E^{+}, E^{-}\right]$of subsets of $M$ such that the union $B=E^{0} \cup \sigma E^{0} \cup E^{+} \cup E^{-}$is disjoint, $B$ is a basis of $M$ and the two conditions

(i) $\sigma e=e$ for $e \in E^{+}$,

(ii) $\sigma e=-e$ for $e \in E^{-}$

hold. We write $B=\left[E^{0}, E^{+}, E^{-}\right]$for short, regarding the triple as a subset of $M$. Note that the structure of $\mathbb{Z}[G]$-modules where $G$ is an abelian group is well known [4] and therefore the existence of a $\sigma$-basis for every $\mathbb{Z}[\sigma]$-module is obvious. See also Remark 2.8 .

In the following we state some results about $\sigma$-bases and give algorithms how to construct them. The proofs of the lemmata and the verification of the algorithms are straightforward.

Lemma 2.1. The set $(1-\sigma) E^{0} \cup E^{-}$is a basis of $\operatorname{ker}_{M}(1+\sigma)$ and the set $(1+\sigma) E^{0} \cup E^{+}$is a basis of $\operatorname{ker}_{M}(1-\sigma)$.

Lemma 2.2. The set $E^{-}$generates the torsion subgroup of $M /(1-\sigma) M$, that is, the torsion elements are of the form $\sum_{e \in E^{-}} \delta_{e} e+(1-\sigma) M$ with $\delta_{e} \in\{0,1\}$. Similarly, $E^{+}$generates the torsion subgroup of $M /(1+\sigma) M$.

REMARK 2.3. The values $m^{+}=m^{+}(M)=\left|E^{+}\right|$and $m^{-}=m^{-}(M)=$ $\left|E^{-}\right|$are invariants of $M$. They are independent of a special choice of $E^{+}$or $E^{-}$. More concretely, $m^{+}$is the dimension of the $\mathbb{F}_{2}$-vector space $H^{0}(\sigma, M)$, and $m^{-}$is the dimension of $H^{1}(\sigma, M)$. 
Algorithm 2.4. Let $C=\left[F^{0}, F^{+}, F^{-}\right]$be a $\sigma$-basis of another module $L$. Then $\left[G^{0}, G^{+}, G^{-}\right] \subseteq M \times L$ with

$$
\begin{aligned}
G^{0} & =\left(E^{0} \times C\right) \cup\left(E^{+} \times F^{0}\right) \cup\left(E^{-} \times F^{0}\right), \\
G^{+} & =\left(E^{+} \times F^{+}\right) \cup\left(E^{-} \times F^{-}\right), \\
G^{-} & =\left(E^{+} \times F^{-}\right) \cup\left(E^{-} \times F^{+}\right)
\end{aligned}
$$

induces a $\sigma$-basis of $M \otimes L$.

A direct consequence of Algorithm 2.4 is the following lemma.

Lemma 2.5. For $i=1, \ldots, r$ let $M_{i}$ be a module with a $\sigma$-basis $\left[E_{i}^{0}, \emptyset, E_{i}^{-}\right]$. The repeated application of Algorithm 2.4 leads to a $\sigma$-basis $\left[F^{0}, F^{+}, F^{-}\right]$of $\bigotimes_{i=1}^{r} M_{i}$ with

$$
\begin{cases}F^{+}=E_{1}^{-} \times \ldots \times E_{r}^{-} \quad \text { and } \quad F^{-}=\emptyset & \text { if } r \text { is even } \\ F^{+}=\emptyset \text { and } \quad F^{-}=E_{1}^{-} \times \ldots \times E_{r}^{-} & \text {if } r \text { is odd } .\end{cases}
$$

Algorithm 2.6. Given sets $B, C$ and an exact sequence of modules

$$
0 \rightarrow M \rightarrow L \rightarrow K \rightarrow 0
$$

such that $B$ is a $\sigma$-basis of $M$ and $\emptyset \neq C \subseteq L$ induces a $\sigma$-basis of $K$. We show how to construct sets $B^{\prime}$ and $C^{\prime}$ such that

(i) $B^{\prime}$ is a $\sigma$-basis of $M^{\prime}=\left\langle B^{\prime}\right\rangle$,

(ii) $C^{\prime}$ is a $\sigma$-basis of $K^{\prime}=\left\langle C^{\prime}\right\rangle$,

(iii) the sequence $0 \rightarrow M^{\prime} \rightarrow L \rightarrow K^{\prime} \rightarrow 0$ is exact,

(iv) $\left|C^{\prime}\right|<|C|$.

We write $B=\left[F^{0}, F^{+}, F^{-}\right]$and $C=\left[E^{0}, E^{+}, E^{-}\right]$. Without loss of generality we assume that $M$ is a submodule of $L$. If $E^{0} \neq \emptyset$ we define $B^{\prime}=\left[F^{0} \cup E^{0}, F^{+}, F^{-}\right]$and $C^{\prime}=\left[\emptyset, E^{+}, E^{-}\right]$. Otherwise we choose an $e \in C$ and set $C^{\prime}=C \backslash\{e\}$. The basis $B^{\prime}=\left[G^{0}, G^{+}, G^{-}\right]$is now defined through the following cases.

$c \in E^{+}$: We have $c-\sigma c \in \operatorname{ker}_{M}(1+\sigma) \subseteq M$. Therefore, by Lemma 2.1, we find $a \in M$ and $F^{\prime} \subseteq F^{-}$such that $c-\sigma c=(1-\sigma) a+\sum_{b \in F^{\prime}} b$. Let $f=c-a$.

- If $F^{\prime}=\emptyset$ we define $G^{0}=F^{0}, G^{+}=F^{+} \cup\{f\}$ and $G^{-}=F^{-}$.

- If $F^{\prime} \neq \emptyset$ we choose $f^{\prime} \in F^{\prime}$ and define $G^{0}=F^{0} \cup\{f\}, G^{+}=F^{+}$and $G^{-}=F^{-} \backslash\left\{f^{\prime}\right\}$.

$c \in E^{-}$: Analogously to the first case we have $a \in M$ and $F^{\prime} \subseteq F^{+}$such that $c+\sigma c=(1+\sigma) a+\sum_{b \in F^{\prime}} b$. Let $f=c-a$.

- If $F^{\prime}=\emptyset$ we define $G^{0}=F^{0}, G^{+}=F^{+}$and $G^{-}=F^{-} \cup\{f\}$.

- If $F^{\prime} \neq \emptyset$ we choose $f^{\prime} \in F^{\prime}$ and define $G^{0}=F^{0} \cup\{f\}, G^{+}=F^{+} \backslash\left\{f^{\prime}\right\}$ and $G^{-}=F^{-}$. 
Algorithm 2.7. Given sets $B, C$ and an exact sequence $0 \rightarrow M \rightarrow L \rightarrow$ $K \rightarrow 0$ such that $B$ is a $\sigma$-basis of $M$ and $C$ is a $\sigma$-basis of $K$ we construct a $\sigma$-basis of $L$ by successively applying Algorithm 2.6 until we have an exact sequence $0 \rightarrow M^{\prime} \rightarrow L \rightarrow 0 \rightarrow 0$ with a $\sigma$-basis of $M^{\prime} \cong L$.

REMARK 2.8. Algorithm 2.7 gives an easy proof that every free module $L$ has a $\sigma$-basis:

Let $M=\{a \in L: \sigma a=a\}$ and $B$ be a basis of $M$. Obviously, $[\emptyset, B, \emptyset]$ is a $\sigma$-basis of $M$ and any set $C \subseteq L$ which induces a basis of $L / M$ induces a $\sigma$-basis $[\emptyset, \emptyset, C]$ of $L / M$ (note that $L / M$ is free). Algorithm 2.7 for the exact sequence

$$
0 \rightarrow M \rightarrow L \rightarrow L / M \rightarrow 0
$$

leads then to a $\sigma$-basis of $L$.

We recall the definition of a $M \mathcal{E}$ n-system as introduced in [2]. In the following let $\Delta$ be a finite, partially ordered indexing set. For a set $A$ we denote by $\langle A\rangle$ the module generated by $A$.

Definition 2.9. For every $d \in \Delta$ let $M_{d}$ be a module, $\mathcal{E}_{d}$ a $\sigma$-invariant subset of $M_{d}$ and $\mathrm{n}_{d}: \mathcal{E}_{d} \rightarrow \bigoplus_{t<d} M_{t}$ a mapping. We call such a system of triples $\left(M_{d}, \mathcal{E}_{d}, \mathrm{n}_{d}\right)_{d \in \Delta}$ a $M \mathcal{E}$-system.

Let $N_{d}^{\prime}=\bigoplus_{t<d} M_{t}$ and $Q_{d}^{\prime}=\sum_{t<d}\left\langle r+\mathrm{n}_{t}(r): r \in \mathcal{E}_{t}\right\rangle \leq N_{d}^{\prime}$. The $M \mathcal{E}$-system $\Gamma$ is combinable if the mappings $\mathrm{n}_{d}$ can be extended to $\sigma$ homomorphisms

$$
\overline{\mathrm{n}}_{d}:\left\langle\mathcal{E}_{d}\right\rangle \rightarrow N_{d}^{\prime} / Q_{d}^{\prime} .
$$

In this case $\Gamma$ defines the module $\mathcal{L}=N / Q$ with $N=\bigoplus_{t \in \Delta} M_{t}$ and $Q=$ $\sum_{t \in \Delta}\left\langle r+\mathrm{n}_{t}(r): r \in \mathcal{E}_{t}\right\rangle$. We call $\mathcal{L}$ the combination of $\Gamma$.

Algorithm 2.10. We show how to construct explicitly a $\sigma$-basis of $\mathcal{L}$ from $\sigma$-bases of the modules $M_{d} /\left\langle\mathcal{E}_{d}\right\rangle$.

We complete the ordering of $\Delta$ and may assume $\Delta=\{1, \ldots, n\}$. For $i \in \Delta$ let $N_{i}=M_{1} \oplus \ldots \oplus M_{i}$ and

$$
Q_{i}=\sum_{j=1}^{i}\left\langle r+\mathrm{n}_{j}(r): r \in \mathcal{E}_{j}\right\rangle \leq N_{i} .
$$

From [2] we know that the sequences

$$
0 \rightarrow N_{i-1} / Q_{i-1} \rightarrow N_{i} / Q_{i} \rightarrow M_{i} /\left\langle\mathcal{E}_{i}\right\rangle \rightarrow 0
$$

(with $N_{0}=Q_{0}=\{0\}$ ) are exact. Starting with $i=1$ and using Algorithm 2.7 we successively construct a $\sigma$-basis of $N_{i} / Q_{i}$ from $\sigma$-bases of $M_{i} /\left\langle\mathcal{E}_{i}\right\rangle$ and $N_{i-1} / Q_{i-1}$. The algorithm ends for $i=n$ and we obtain a $\sigma$-basis of $\mathcal{L}=N_{n} / Q_{n}$. 
3. The cyclotomic system. In this section we recall the definitions and the properties of the cyclotomic module and the cyclotomic system. More details and proofs of the lemmata can be found in [2]. For a subset $S$ of a module $M$ we write $\Sigma S$ for $\sum_{s \in S} s$. Further, let $G_{d}=\{1 \leq a<d:(a, d)=$ $1\}$ and for each $d>2$ we fix a subset $H_{d} \subseteq G_{d}$ such that for all $a \in G_{d}$ either $a \in H_{d}$ or $d-a \in H_{d}$. For instance we can choose $H_{d}=\{1, \ldots,\lfloor d / 2\rfloor\} \cap G_{d}$.

Definition 3.1. For $n \in \mathbb{N}$ we define the cyclotomic module $Z(n)$ as follows:

If $n=p$ prime then $Z(p)=\left\langle G_{p}\right\rangle /\left\langle\Sigma G_{p}\right\rangle$.

If $n=q=p^{\alpha}$ with $\alpha>1$ then

$$
Z(q)=\left\langle G_{q / p}\right\rangle \otimes\left\langle A_{p}\right\rangle /\left\langle\Sigma A_{p}\right\rangle \quad \text { with } A_{p}=\{0, \ldots, p-1\} .
$$

If $n=q_{1} \ldots q_{r}$ where the $q_{i}$ are powers of distinct primes then

$$
Z(n)=Z\left(q_{1}\right) \otimes \ldots \otimes Z\left(q_{r}\right) .
$$

We define the operation of $\sigma$ on $b \in G_{d}$ by $\sigma b=d-b$ and on $a \in A_{p}$ by $\sigma a=p-1-a$. By this $Z(n)$ becomes a module with an involution $\sigma$.

LEMmA 3.2. We have $Z(n) \cong\left\langle G_{n}\right\rangle / R_{n}$ where the submodule $R_{n}$ of $\left\langle G_{n}\right\rangle$ is generated by $\mathcal{E}_{n}=\left\{s(n, p, a): p \mid n\right.$ with $p$ prime, $\left.a \in G_{n / p}\right\}$ with

$$
s(n, p, a)=\Sigma\left\{x \in G_{n}: x \equiv a \bmod (n / p)\right\} .
$$

REMARK 3.3. The isomorphism in Lemma 3.2 can be described explicitly: We order the prime factors $p_{i}$ of $n$ such that $n=p_{1}^{\alpha_{1}} \ldots p_{t}^{\alpha_{t}} p_{t+1} \ldots p_{r}$ with $\alpha_{i}>1$ for $i=1, \ldots, t$. Further, we set $q_{i}=p_{i}^{\alpha_{i}}$ if $i=1, \ldots, t$ and $q_{i}=p_{i}$ otherwise. Let

$$
S=G_{q_{1} / p_{1}} \times A_{p_{1}} \times \ldots \times G_{q_{t} / p_{t}} \times A_{p_{t}} \times G_{p_{t+1}} \times \ldots \times G_{p_{r}} .
$$

It is obvious, by the definition of the tensor product, that $Z(n)$ is isomorphic to $\langle S\rangle$ modulo suitable relations. We obtain $S \cong G_{n}$ by a combination of isomorphisms $\xi_{i}$ and $\eta$ in the following way:

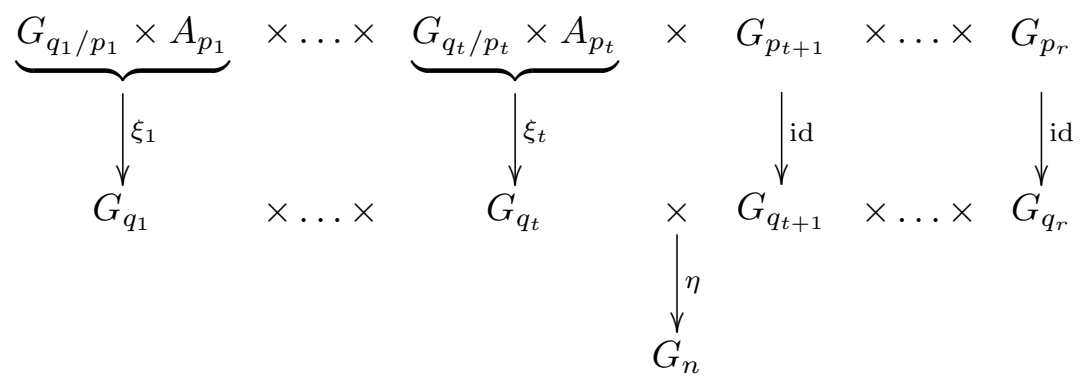

The maps $\xi_{i}$ for $i=1, \ldots, t$ are explicitly given by $\xi_{i}(b, a)=a p_{i}^{\alpha_{i}-1}+b$. The map $\eta$ is defined by the Chinese remainder theorem, that is, $\eta^{-1}$ is the map $a \mapsto a \bmod q_{i}$ in each component $G_{q_{i}}, i=1, \ldots, r$. 
Definition 3.4. For $d \in \mathbb{N}$ let $M_{d}=\left\langle G_{d}\right\rangle$. If $d$ is not a prime we denote by $\mathcal{E}_{d} \subseteq M_{d}$ the set of the sums $s(d, p, a)$ as in Lemma 3.2. For $d$ prime we define $\mathcal{E}_{d}=\emptyset$. On $\mathcal{E}_{d}$ we define the mapping

$$
\mathrm{n}_{d}: \mathcal{E}_{d} \rightarrow \bigoplus_{t \mid d, t \neq d} M_{t}, \quad s(d, p, a) \mapsto \begin{cases}-[d / p ; a] & \text { if } p^{2} \mid d, \\ {\left[d / p ; p^{-1} a\right]-[d / p ; a]} & \text { if } p^{2} \nmid d,\end{cases}
$$

where $[m ; x]$ denotes $y \in G_{m}$ with $x \equiv y \bmod m$.

For $n \in \mathbb{N}$ we call the $M \mathcal{E}$ n-system $\Gamma(n)=\left(M_{d}, \mathcal{E}_{d}, \mathrm{n}_{d}\right)_{d \mid n}$ the $n$th cyclotomic system.

Lemma 3.5. (a) $\Gamma(n)$ is combinable. Let $\mathcal{L}(n)$ be the combination of $\Gamma(n)$.

(b) Let $n>2$ and $r$ be the number of prime factors of $n$. Then

(i) $H^{0}(\sigma, \mathcal{L}(n)) \cong \begin{cases}\mathbb{F}_{2}^{2^{r-1}-1} & \text { if } n \neq 2 \bmod 4, \\ \mathbb{F}_{2}^{2^{r-2}} & \text { if } n \equiv 2 \bmod 4 .\end{cases}$

(ii) $H^{1}(\sigma, \mathcal{L}(n)) \cong \begin{cases}\mathbb{F}_{2}^{2^{r-1}-r} & \text { if } n \neq \equiv \bmod 4, \\ \mathbb{F}_{2}^{2^{r-2}-r+1} & \text { if } n \equiv 2 \bmod 4 .\end{cases}$

(c) The sequences in (8) for the cyclotomic system split over $\sigma$ (with one exception for $n=4)$. This has in particular the consequence that in Algorithm 2.6 only the case $F^{\prime}=\emptyset$ occurs.

(d) The sequence

$$
0 \rightarrow T(n) \rightarrow \mathcal{L}(n) /(1-\sigma) \mathcal{L}(n) \stackrel{\bar{\mu}}{\rightarrow} D^{(n)} \rightarrow 1
$$

where $T(n)$ is the torsion subgroup of $\mathcal{L}(n) /(1-\sigma) \mathcal{L}(n)$, is exact. The homomorphism $\bar{\mu}$ is defined by the maps $\mu_{d}: G_{d} \rightarrow D^{(n)}, a \mapsto 1-\varepsilon_{d}^{a}$, for $d \mid n$ where $\varepsilon_{d}$ is a primitive dth root of unity such that $\varepsilon_{d}=\varepsilon_{t}^{t / d}$ whenever $d \mid t$.

(e) Let $K^{(n)}=\prod_{d \mid n, d \neq n} D^{(d)}$. We call $\widehat{D^{(n)}}=D^{(n)} / K^{(n)}$ the group of $n$th relative cyclotomic numbers. Then for $n \neq 4$ we have an exact sequence

$$
0 \rightarrow \widehat{T}(n) \rightarrow Y(n) /(1-\sigma) Y(n) \stackrel{\bar{\mu}}{\rightarrow} \widehat{D^{(n)}} \rightarrow 1
$$

where $Y(n)=M_{n} /\left\langle\mathcal{E}_{n}\right\rangle$ with $M_{n}$ and $\mathcal{E}_{n}$ as in Definition 3.4.

Table 1. Some examples of $\sigma$-bases

\begin{tabular}{c|c}
\hline Module & $\sigma$-basis \\
\hline$\left\langle G_{2}\right\rangle$ & {$[\emptyset,\{1\}, \emptyset]$} \\
$\left\langle G_{p}\right\rangle, p \neq 2, p$ prime & {$\left[H_{p}, \emptyset, \emptyset\right]$} \\
$Z(2)$ & {$[\emptyset, \emptyset, \emptyset]$} \\
$Z(p), p \neq 2, p$ prime & {$\left[\{2, \ldots,(p-1) / 2\}, \emptyset, \Sigma H_{p}\right]$} \\
$Z(4)$ & {$[\emptyset, \emptyset,\{(1,0)\}]$} \\
$Z(q), q=p^{\alpha}, \alpha>1$, & {$\left[\left\{(b, a): b \in H_{q / p}, 1 \leq a<p\right\}, \emptyset, \emptyset\right]$} \\
$p$ prime,$q \neq 4$ & \\
\hline
\end{tabular}


Note that using Algorithm 2.10 we can construct a $\sigma$-basis of $\mathcal{L}(n)$ from $\sigma$-bases of the modules $Y(d)$ for $d \mid n$. Using the isomorphism of Lemma 3.2 and Algorithm 2.4 we are able to construct $\sigma$-bases of the $Y(d)$ from the $\sigma$-bases from Table 1 .

4. Ennola relations. In this section we have a closer look at the exact sequence (13). Note that the norm relations as in (1) are given implicitly in (13) as the relations of the form $s(d, p, a)+\mathrm{n}_{d}(s(d, p, a))$ in the definition of $\mathcal{L}(n)$. The relations $(2)$ correspond to factoring by $(1-\sigma) \mathcal{L}(n)$.

Algorithm 4.1. We construct Ennola relations for $D^{(n)}$ performing the following steps.

1. With the algorithms of Section 2 compute a $\sigma$-basis $\left[E^{0}, E^{+}, E^{-}\right]$of $\mathcal{L}(n)$.

2. Each element of $E^{-}$gives by Lemma 2.2 a nontrivial element of $T(n)$ which is a relation in $D^{(n)}$ via the mapping $\bar{\mu}$ in (13).

Note that Algorithm 4.1 has been implemented in a $\mathrm{C}++$ extension of the computer algebra system SIMATH [9]. A description of the implementation and examples can be found in [3].

The smallest number where $T(n)$ is nontrivial is $n=60$. An Ennola relation in $D^{(60)}$ is

$$
\left(1-\varepsilon_{60}\right)\left(1-\varepsilon_{60}^{37}\right)\left(1-\varepsilon_{20}^{17}\right)^{-1}\left(1-\varepsilon_{15}\right)^{-1}\left(1-\varepsilon_{12}\right)^{-1}=\varepsilon_{15}
$$

where $\varepsilon_{d}=\varepsilon_{n}^{n / d}$ for $d \mid n$.

TheOREM 4.2. For $n>2$ let $r$ be the number of prime factors of $n$ and

$$
c= \begin{cases}2^{r-1}-r & \text { for } n \neq \equiv 2 \bmod 4, \\ 2^{r-2}-r+1 & \text { for } n \equiv 2 \bmod 4 .\end{cases}
$$

Then there exist in $D^{(n)}$ exactly $2^{c}-1$ different Ennola relations which are generated by c relations. More exactly, we have elements $d_{1}, \ldots, d_{c}$ in the free group generated by the set $\left\{1-\varepsilon_{d}^{a}: d \mid n, a \in G_{d}\right\}$ such that the Ennola relations are of the form $\prod_{i=1}^{c} d_{i}^{\delta_{i}}$ with $\delta_{i} \in\{0,1\}$. The $d_{i}$ for $i=1, \ldots, c$ can be constructed explicitly with Algorithm 4.1.

Pr o o f. Let $\left[E^{0}, E^{+}, E^{-}\right]$be a $\sigma$-basis of $\mathcal{L}(n)$. Then we have $c=\left|E^{-}\right|$by Lemma 2.2. Because $\left|E^{-}\right|$is the dimension of $H^{1}(\sigma, \mathcal{L}(n))$ the claim follows from Lemma 3.5.

As noted in Lemma 3.5, we see that in the process of constructing a $\sigma$-basis of $\mathcal{L}(n)$ in Algorithm 2.6 for $n>4$ always the subcase $F^{\prime}=\emptyset$ happens. So we can force $T(d) \subseteq T(n)$ for $d \mid n$ and get immediately the following corollary. 
Corollary 4.3. Let $D^{(\infty)}=\bigcup_{d \in \mathbb{N}} D^{(d)}$. Then there exist elements $d_{1}, d_{2}, \ldots$ in the free group generated by the set $\left\{1-\varepsilon_{d}^{a}: d \in \mathbb{N}, a \in G_{d}\right\}$ such that the Ennola relations are of the form $\prod_{i \in I} d_{i}^{\delta_{i}}$ with $\delta_{i} \in\{0,1\}$ where $I$ is a finite subset of $\mathbb{N}$. The $d_{i}$ for $i \in \mathbb{N}$ can be constructed explicitly with Algorithm 4.1.

A closer look at the explicit structure of the Ennola relations is taken in the next section.

5. Ennola relations in $\widehat{D^{(n)}}$. Let $n \neq 4$. We write $n=q_{1} \ldots q_{r}$ where the $q_{i}$ are powers of distinct primes.

LEMMA 5.1. The torsion group $\widehat{T}(n)$ in (14) is isomorphic to $\mathbb{Z} / 2 \mathbb{Z}$ in the following two cases:

$$
\begin{aligned}
& r \text { odd, } r \neq 1 \text { and } n=u, \\
& r \text { odd, } r \neq 1 \text { and } n=4 u,
\end{aligned}
$$

where $u$ is odd and square free. In all other cases $\widehat{T}(n)$ is trivial.

Proof. By Lemma 2.2, $\widehat{T}(n)$ is generated by the set $E^{-}$where $\left[E^{0}, E^{+}, E^{-}\right]$is a $\sigma$-basis of $Y(n)$. If $n=p$ is a prime then $Y(p)=\left\langle G_{p}\right\rangle$ and Table 1 shows $E^{-}=\emptyset$. In the other cases $Y(n) \cong Z\left(q_{1}\right) \otimes \ldots \otimes Z\left(q_{r}\right)$ by Lemma 3.2. Here, Lemma 2.5 shows how we obtain a $\sigma$-basis of $Z(n)$ from $\sigma$-bases of the $Z\left(q_{i}\right)$ which are given in Table 1 . Note that $E^{-}=\emptyset$ if for at least one of the $\sigma$-bases $\left[E_{i}^{\emptyset}, \emptyset, E_{i}^{-}\right]$of the modules $Z\left(q_{i}\right)$ we have $E_{i}^{-}=\emptyset$.

THEOREM 5.2. The relations in the group of relative cyclotomic numbers $\widehat{D^{(n)}}=D^{(n)} / K^{(n)}$ are the obvious relations (as in (1) and $(2)$ ) and in the cases of (17) Ennola relations. The latter are given implicitly by the set $\widehat{T}(n)$ in (14) and can be explicitly written as

$$
\prod_{a \in V_{n}}\left(1-\varepsilon_{n}^{a}\right) \in K^{(n)}
$$

where $V_{n} \cong H_{q_{1}} \times \ldots \times H_{q_{r}}$ via the isomorphism $\eta$ given in (11).

P r o of. Lemma 5.1 describes $\widehat{T}(n)$ and how it can be constructed explicitly from a $\sigma$-basis of the modules $Z\left(q_{i}\right)$. The isomorphism in Remark 3.3 shows (18).

6. Stickelberger elements. Let $I_{n}$ be the fractional ideal in the group ring $\mathbb{Q}\left[G_{n}\right]$ which is generated by the Stickelberger elements

$$
\theta(a)=\sum_{\tau \in G_{n}}\langle-a \tau / n\rangle \tau^{-1}
$$


and $\omega_{n}=\Sigma G_{n}$ for $n$ odd and $\omega_{n}=\frac{1}{2} \Sigma G_{n}$ for $n$ even. There is a strong connection between the relations of cyclotomic numbers and the relations between Stickelberger elements (see [6], [8]). In our context, in analogy to the exact sequence (13), the sequence

$$
0 \rightarrow T^{\prime}(n) \rightarrow \mathcal{L}(n) /(1+\sigma) \mathcal{L}(n) \stackrel{\bar{\nu}}{\rightarrow} I_{n} /\left\langle\omega_{n}\right\rangle \rightarrow 0
$$

is exact where the homomorphism $\bar{\nu}$ is defined by the maps $\nu_{d}: G_{d} \rightarrow I_{n}$, $a \mapsto \theta(a n / d)$.

This situation can be handled as in the case of cyclotomic numbers. We call the elements of $T^{\prime}(n)$ Ennola relations.

Algorithm 6.1. We construct Ennola relations for $I_{n} /\left\langle\omega_{n}\right\rangle$ performing the following steps.

1. As in Algorithm 4.1, compute a $\sigma$-basis $\left[E^{0}, E^{+}, E^{-}\right]$of $\mathcal{L}(n)$.

2. Each element of $E^{+}$leads to a nontrivial element of $T^{\prime}(n)$ which is a relation in $I_{n} /\left\langle\omega_{n}\right\rangle$ via the mapping $\bar{\nu}$ in $(20)$.

THEOREM 6.2. For $n>2$ let $r$ be the number of prime factors of $n$ and

$$
c^{\prime}= \begin{cases}2^{r-1}-1 & \text { for } n \neq \equiv 2 \bmod 4, \\ 2^{r-2} & \text { for } n \equiv 2 \bmod 4 .\end{cases}
$$

Then there exist in $I_{n} /\left\langle\omega_{n}\right\rangle$ exactly $2^{c^{\prime}}$ different Ennola relations which are generated by $c^{\prime}$ different relations. These relations can be constructed explicitly with Algorithm 6.1.

As an example, we show an Ennola relation for $n=15$. We have

$$
\theta(1)+\theta(7)-\theta(3)-\theta(5)=0 .
$$

Remark 6.3. There is no canonical definition of a "relative Stickelberger ideal" as there is one for relative cyclotomic numbers. However, we can investigate the torsion group $\widehat{T^{\prime}}(n)$ of $Y(n) /(1+\sigma) Y(n)$ and get in analogy to Lemma 5.1 the following result:

The torsion group $\widehat{T^{\prime}}(n)$ is isomorphic to $\mathbb{Z} / 2 \mathbb{Z}$ in the following two cases:

$$
\begin{aligned}
& r \text { even and } n=u, \\
& r \text { even and } n=4 u,
\end{aligned}
$$

where $u$ is odd and square free. In all other cases $\widehat{T^{\prime}}(n)$ is trivial.

\section{References}

[1] H. Bass, Generators and relations for cyclotomic units, Nagoya Math. J. 27 (1966), 401-407.

[2] M. Conrad, Construction of bases for the group of cyclotomic units, J. Number Theory, to appear. 
[3] M. Conrad, Basen von Moduln mit Anwendung auf Kreiseinheiten und Stickelbergerelemente, Dissertation an der Universität des Saarlandes, Saarbrücken, 1997.

[4] C. W. Curtis and I. Reiner, Representation Theory of Finite Groups and Associative Algebras, Interscience, New York, 1966.

[5] V. Ennola, On relations between cyclotomic units, J. Number Theory 4 (1972), $236-247$.

[6] R. Kučera, On bases of the Stickelberger ideal and of the group of circular units of a cyclotomic field, ibid. 40 (1992), 284-316.

[7] K. Ramachandra, On the units of cyclotomic fields, Acta Arith. 12 (1966), 165173 .

[8] C.-G. Schmidt, Die Relationsfaktorgruppen von Stickelberger-Elementen und Kreiszahlen, J. Reine Angew. Math. 315 (1980), 60-72.

[9] SIMATH, Ein Computeralgebrasystem für algorithmische Zahlentheorie, http:// simath.math.uni-sb.de.

[10] W. Sinnott, On the Stickelberger ideal and the circular units of a cyclotomic field, Ann. of Math. 108 (1978), 107-134.

[11] L. C. Washington, Introduction to Cyclotomic Fields, Springer, New York, 1997.

Gärtnerstraße 5

D-66117 Saarbrücken

Germany

E-mail: marc@math.uni-sb.de

Web: http://emmy.math.uni-sb.de/ marc

Received on 22.3.1999

and in revised form on 28.10.1999 